\title{
Licenciatura en Pedagogía de la Madre Tierra, etnomatemática y formación de profesores
}

\section{Degree in pedagogy of Mother Earth, Etnomathematics and teacher training}

\author{
Carolina Tamayo Osorio ${ }^{1}$ \\ https://orcid.org/0000-0002-8478-7845
}

\begin{abstract}
Resumen: El objetivo de este artículo es presentar los efectos de la inclusión de la Etnomatemática como curso en el programa de formación de profesores indígenas de la Licenciatura en Pedagogía de la Madre Tierra (LPMT) de la Universidad de Antioquia, Colombia. Partimos de la experiencia de dos profesores indígenas que enseñan matemáticas en una comunidad de Alto Caimán, que se formaron en la LPMT. Evidenciamos que la inclusión de la Etnomatemática como uno de los ejes centrales ha posibilitado movimientos de desconstrucción de la colonialidad del saber desde la universidad para, con y desde las comunidades indígenas, cuestionando la Matemática escolar de carácter eurocéntrico, para hablar de matemáticas como conjuntos de acciones normativamente orientadas con fines inequívocos, esto es, como juegos de lenguaje.
\end{abstract}

Palabras-clave: Educación indígena. Etnomatemática. Enseñanza de matemática. Educación superior. Colombia.

\begin{abstract}
The objective of this article is to present the effects of the inclusion of Ethnomathematics as a course in the training program for indigenous teachers of the Bachelor of Pedagogy of Mother Earth (BPME) of the University of Antioquia, Colombia. We start from the experience of two indigenous teachers who teach mathematics in an Alto Cayman community, who were trained in the BPME. We show that the inclusion of Ethnomathematics as one of the central axes has enabled movements of deconstruction of the coloniality of knowledge from the university to, with and from indigenous communities to problematize school Mathematics and their Eurocentric character, to speak in Mathematics as normatively oriented sets of actions with unequivocal aims, that is, as language games.
\end{abstract}

Keywords: Indigenous education. Etnomathematics. Mathematics teaching. Higher education. Colombia.

\footnotetext{
${ }^{1}$ Universidade Federal de São Carlos (UFSCar), Centro de Educação e Ciências Humanas, Departamento de Metodologia de Ensino, São Carlos, SP, Brasil. E-mail: <carolina.tamayoo@udea.edu.co>.
} 


\section{Introducción}

Colombia tiene, según el censo de 2005, una población indígena de 1.392.623, de los cuales 770.633 viven en sus resguardos ${ }^{2}$ (COLOMBIA, 2005), cantidad que corresponde al $3,43 \%$ de la población del país y que se caracteriza por ser ampliamente diversa. Esta población está distribuida entre más de 80 etnias hablantes de 65 lenguas indígenas aproximadamente, y viven en comunidades rurales y selváticas, en las cuales el acceso a la educación es complejo, aun considerando la existencia de políticas públicas que buscan revertir tal situación. Aún así, la presencia de estudiantes indígenas en las universidades colombianas es un fenómeno de las últimas décadas, aun así, tiende a crecer rápidamente, a pesar de que se "estima que solo el 0,6\% de los estudiantes matriculados en todas las modalidades de Educación Superior (técnica profesional, tecnológica universitaria) es indígena, lo cual refleja desigualdad" (PANCHO, 2004, p. 107).

Es importante señalar que una de las principales consignas del movimiento indígena nacional, desde los años 1970, fue la formación de profesores indígenas bilingües, con la finalidad de que se ellos pudiesen organizar y gestionar la educación escolar en sus comunidades. De allí surgen diversas discusiones sobre interculturalidad, bilingüismo y organización curricular de las escuelas en territorios indígenas que se han configurado en el país. Estas discusiones son efecto de que la educación para y de los pueblos indígenas en Colombia se ha constituido, ante todo, en un proyecto político que tiene por objetivo la formación de líderes sociales y políticos, la pervivencia cultural y el camino para materializar el plan de vida de cada pueblo, aun así, como destaca Sierra (2004, p. 91),

[...] contrario a lo que se pudiera esperar, dado el carácter colectivo y solidario de la cultura indígena, muchos de los y las bachilleres indígenas que deciden continuar sus estudios universitarios lo vienen haciendo más por decisión personal que por políticas claras de la comunidad o la organización indígena; en algunas ocasiones, contando si con el apoyo y estímulo de la familia y la comunidad, pero sin una articulación real a los procesos organizativos o comunitarios definidos, y en ocasiones en contravía. No se debe desconocer, sin embargo, esa fuerza personal interna que da comienzo al complejo proceso de buscar información, conseguir formularios de inscripción, seleccionar la carrera, tramitar recursos locales, regionales o nacionales, intentarlo una y otra vez si es necesario, y desplazarse a la ciudad en la cual se localiza la universidad, para asumir todo el riesgo de lo desconocido.

A pesar de las limitaciones que enfrentan los jóvenes indígenas, dentro de las cuales está el uso del español como lengua dominante en las pruebas de Estado y en las universidades,

\footnotetext{
${ }^{2}$ Actualmente hay 737 resguardos legalmente constituidos. El DANE certifica la población de 796, ubicados en 234 municipios y en 27 departamentos.
} 
es importante resaltar que muchos de ellos poseen trayectorias de trabajo comunitario y organizativo que les posibilita sobreponerse a la dominación epistémica occidental en sus procesos de formación como profesores, además, ellos reconocen que la condición de indígenas y de profesores/líderes representa un sello de identidad en las escuelas de sus comunidades. Tal postura posibilita la lucha y reivindicación por el acceso a unas condiciones jurídicas especiales y unas demarcaciones culturales leídas desde los lenguajes, las estéticas, la corporeidad, las formas de organización social y de representación del mundo.

Es importante resaltar que a partir de la Constitución Política de 1991, y desde sus artículos 7, 8, 10, 19, 68 y 72 (COLOMBIA, 1991) reglamentados en el capítulo III de la ley 115/94 (COLOMBIA, 1994) y del Decreto 804/95 del Ministerio de Educación Nacional (COLOMBIA, 1995) - aunque el Decreto 1142 de 1978 (COLOMBIA, 1978), es la primera disposición que reconoce oficialmente la educación indígena - se propone garantizar los derechos educativos para grupos étnicos, al mismo tiempo que dar orientaciones al Estado para implementar programas educativos apropiados y especializados para estos grupos del país. Para tal fin, fue adoptada la política de Etnoeducación que legitima el derecho de los grupos étnicos para direccionar, gestionar y orientar sus Proyectos Educativos Comunitarios (PEC). Es importante señalar que para las comunidades indígenas colombianas los PEC son una estrategia que contribuyen al desarrollo del Plan de vida de cada pueblo, pues están centrados

[...] en su territorialidad, organización, cultura y jurisdicción propia. Recogen y proponen alternativas a las problemáticas productivas, eco-ambientales, sociales, culturales, entre otras. El PEC armoniza y fortalece los procesos educativos de los pueblos indígenas, los procesos educativos cotidianos, permanentes y la educación escolarizada, la cual es una parte que se inserta y articula en el marco de los lineamientos del Sistema Educativo Indígena Propio. Los espacios de familia, de ritualidad, de fiesta, de trabajo comunitario, de formación política y organizativa, de lugares sagrados, de encuentro e intercambio de saberes, de concertación, son escenarios de los aprendizajes con sus formas pedagógicas propias. (ORGANIZACIÓN NACIONAL DE LOS PUEBLOS INDÍGENAS DE LA AMAZONIA COLOMBIANA, 2017).

Así, en Colombia, la Etnoeducación $n^{3}$ constituye una referencia obligada en el análisis de las políticas educativas para los campesinos, afrodescendientes e indígenas, como ha sido documentado por Castillo y Rojas (2005), aunque en muchos casos esa propuesta se aleja de los planes de vida comunitarios y de los PEC. Es importante señalar que el surgimiento de la Etnoeducación hace parte de la historia de una lucha política por una educación diferenciada para las poblaciones étnicas, rechazando las formas de escolarización que les fueron impuestas durante los siglos XVI al XVIII por la iglesia católica, desde la cual es posible visualizar la compleja relación entre colonialidad y saber pues,

\footnotetext{
${ }^{3}$ Bajo el decreto 804 de Etnoeducación (COLOMBIA, 1995).
} 
La colonialidad incorpora no sólo a las poblaciones; incluye también sus conocimientos. Ni la Europa occidental colonialista, moderna y racional, ni la sociedad criolla blanco/mestiza, reconocieron en sus “otros" la capacidad de producir conocimientos válidos, universales. En consecuencia, definieron lo que deberían saber; primero para salvar sus almas, luego para redimir sus mentes. Desde los primeros momentos del proceso de colonización, los saberes que habrían de ser institucionalizados y aprendidos por indígenas y negros fueron definidos al margen de sus intereses. La escuela, cuando llegó, lo hizo para "incorporar", para conducir el tránsito de estas poblaciones hacia la sociedad "civilizada". (CASTILLO; ROJAS, 2005, p. 138).

La Etnoeducación incluye, por tanto, como plantea Camacho (1999), el cuerpo de conocimientos que sirven de puente a modo de "escuela-frontera", entre la educación tradicional de cada pueblo indígena y las demandas de una economía de mercado; sin embargo, desde nuestro punto de vista, se manifiesta una relación utilitaria de la sabiduría indígena, en la medida en que muchas veces tal sabiduría 'sirve' para traducir y legitimar el conocimiento occidental y de este modo continuar legitimando las relaciones asimétricas de poder incorporadas en la colonialidad del saber.

Lo anterior, está vinculado a la comprensión de que la colonialidad del saber es solo una de las esferas que permite entender la dinámica de la colonialidad como eje estructurador de las relaciones sociales que han permitido el dominio de occidente sobre el resto del mundo, y como Tamayo-Osorio (2017a), señala, la escuela moderna ha legitimado tal imposición, al ser organizada con base en el pensamiento eurocéntrico como la única forma de conocer el mundo, bajo la premisa del saber científico como un saber objetivo, neutral, y universial.

Con la emergencia de estas líneas de pensamientos enraizadas en las epistemologías del sur y producto de las luchas de los movimientos indígenas y afrodescendientes en el contexto colombiano, a partir de 1992, fueron instituidos programas universitarios de Licenciatura en Etnoeducación en la Universidad de la Amazonía, la Universidad del Cauca, la Universidad de la Guajira, la Universidad Tecnológica de Pereira, la Universidad Pontificia Bolivariana (UPB) - en convenio con el Instituto Misionero Antropológico -, la Universidad Nacional Abierta y a Distancia, y la Universidad Mariana (ROJAS, 2008). Entre otras opciones de formación en Educación Superior que también fueron posibles gracias a las alianzas entre organizaciones indígenas y universidades, encontramos la alianza entre la Organización Indígena de Antioquia (OIA) y la Universidad de Antioquia, como lo documenta Sierra (2004), de la que surge el programa de Licenciatura en Pedagogía de la Madre Tierra (LPM) en el cual la Etnomatemática aparece integrada al plan curricular desde el primero semestre hasta el último, como una de las manifestaciones de decolonialidad del saber al interior de las universidades para y con los pueblos indígenas en el eje temático de formación pedagógica desde la interculturalidad.

Durante los últimos siete años hemos venido realizando, con dos profesores indígenas Gunadule formados en la LPM diversos proyectos investigación ${ }^{4}$, por ello es de nuestro

${ }^{4}$ CUÉLLAR LEMOS (2017); CUÉLLAR LEMOS; MARTÍNEZ MONTOYA (2013); TAMAYO-OSORIO (2012, 2017a). 
interés en este artículo, analizar desde una perspectiva sociocultural y decolonial los efectos de la inclusión de la Etnomatemática en el programa de formación de profesores indígenas de la Licenciatura en Pedagogía de la Madre Tierra (LPMT) de la Universidad de Antioquia (Medellín, Colombia). Para esto, nos centraremos en la experiencia de los dos profesores que actualmente enseñan matemática $5^{5}$ en la comunidad Gunadule de Alto Caimán en el Urabá antioqueño y nos remitimos a la Etnomatemática en la perspectiva de D’Ambrosio (1993, 1997, 2008), buscando entender ese discurso como un movimiento de contra-conducta que emerge en el interior de la Matemática (MONTEIRO; MENDES, 2015), para cuestionar discursos naturalizados sobre ella vista como un campo disciplinar, neutro y universal.

Es importante aclarar que los registros y datos que vamos a presentar, son producto de los diálogos que hemos establecido como equipo de trabajo colaborativo, pues consideramos, como plantea Jaramillo (2011, p. 13), que "el país requiere establecer diálogos entre las diferentes comunidades académicas y no académicas, que procuren la comprensión de otras formas de objetivación del conocimiento matemático, respetando los distintos saberes constituidos por los diversos grupos al interior de los mismos", pues

Sin colaboración intercultural en la producción de conocimientos sobre nosotros mismos, en tanto agregados sociales, es imposible comprender nuestra experiencia social. Sin esa colaboración intercultural la comprensión de significativos aspectos históricos, jurídicos, políticos, económicos, sociales, y otros de nuestras sociedades, será siempre sesgada y parcial. Sin tal colaboración, la comprensión partirá de un "como si”. Por esto, la colaboración intercultural en la producción de conocimientos sobre nuestras sociedades es imprescindible. (MATO, 2016, p. 8).

Es importante reconocer investigaciones como las de Blanco Álvarez (2008) ya han venido problematizando la integración de la Etnomatemática en la Etnoeducación, sin embargo, este debate continúa abierto y no es foco de análisis de este trabajo.

\section{La licenciatura en Pedagogía de la Madre Tierra}

La Universidad de Antioquia al proponerse contrarrestar las realidades de los pueblos del departamento de Antioquia firmó un acuerdo académico para la admisión especial de grupos étnicos desde $1983^{6}$, que permitió un gradual incremento de estudiantes indígenas en la universidad. Mediante la firma del Convenio Marco de Cooperación No. 019 de 2004

\footnotetext{
${ }^{5}$ El uso de la palabra matemáticaS hace referencia a la deconstrucción la palabra Matemática en cuanto disciplina académica, inspirándome en el proyecto de extensión matemáticaS desarrollado por el grupo de investigación Educação, Linguagem e Práticas Culturais (PHALA), en colaboración con el Laboratório de Estudos Avançados em Jornalismo (LABJOR) de la Universidade Estadual de Campinas (Unicamp).

${ }^{6}$ Acuerdo Académico del 13 de mayo de 1983, actualizado por el Acuerdo Académico 236 del 30 de octubre de 2002. Disponible en: < https://tinyurl.com/y7e83sfw>. Accedido el: 22 ago. 2018.
} 
con la Organización Indígena de Antioquia ${ }^{7}$ (OIA), se dio paso posteriormente a la creación del Programa de Educación Indígena, adscrito a la Facultad de Educación (Resolución Académica 1752 del 18 de agosto de 2005) (UNIVERSIDAD DE ANTIOQUIA, 2005), con el objetivo de abordar de manera efectiva la deuda histórica de la institución con los pueblos indígenas del departamento y el país.

Este momento se tornó fundamental para la defensa por los derechos indígenas de la región, la protección de sus territorios y cultura, y el logro de una mayor participación en la vida política del país que sigue siendo un proceso complejo y difícil. Para darle continuidad a este y otros proyectos, la Universidad de Antioquia y la OIA firman un nuevo Convenio Marco de Cooperación (No. 042 de 2009) en el que la Licenciatura en Pedagogía de la Madre Tierra se inserta en el Plan de Desarrollo de la Universidad de Antioquia 2006-2016, comprometidos en hacer de la institución una Universidad investigadora, innovadora al servicio de las regiones y del país (UNIVERSIDAD DE ANTIOQUIA, 2006b).

La LPMT se desarrolla mediante una metodología de trabajo en tres sentidos, como lo documentan Green, Sinigui y Rojas (2013):

a) Diálogo intercultural que se realiza en los encuentros formativos regionales, durante un periodo de tiempo de 15 días en la sede central de la Universidad, para que los y las estudiantes de las distintas regiones puedan intercambiar saberes y prácticas;

b) Encuentros zonales que se organiza de acuerdo con la cercanía y afinidad territorial y cultural. Por ejemplo, en el departamento de Antioquia se trabaja en zonas: Urabá Norte, Occidente, Bajo Cauca y Medellín. En cada zona territorial se designa una sede para trabajar que puede ser la de una institución municipal o en una sede dentro de una Comunidad.

c) Encuentros formativos locales, para profundizar en las particularidades de los contextos y avanzar propuestas de solución a problemáticas de las comunidades.

Los encuentros zonales y locales tienen como objetivo un dialogo intracultural, aproximando a los estudiantes a sus propias comunidades, con la finalidad de que los sabios y líderes de los pueblos participen activamente de este proceso de formación. Lo anterior está en sintonía con la propuesta de la LPM, en la que se espera que,

Docentes y líderes deben, además, estar en capacidad de leer e interpretar los desafíos que demanda la interacción con otras culturas, en particular, la presión que viven actualmente los territorios indígenas por parte de sectores económicos muy poderosos a nivel nacional y global. Un maestro o una maestra indígena debe ser entonces puente de diálogo entre los saberes ancestrales y los saberes de la cultura dominante y otras culturas con las cuales interactúa su comunidad... Su campo de acción no es la escuela solamente sino la vida comunitaria, concebida como un contexto educador en sí mismo. (GREEN; SINIGUI; ROJAS, 2013, p. 91).

\footnotetext{
${ }^{7}$ Ha sido una conquista del movimiento indígena que la planeación escolar y en general que todo desarrollo institucional, programas y proyectos que se implementen en sus comunidades se fundamenten en los Planes de Vida. (COLOMBIA, 1995). ${ }^{8}$ En el sentido presentado por Miguel, Vilela y Moura (2012) y problematizado desde la educación indígena por Tamayo-Osorio (2017a).
} 
Visando alcanzar estos objetivos, son brindadas diversas disciplinas, entre las cuales se encuentran los cursos de Etnomatemáticas, que son ofrecidos en el ciclo básico - durante los primeros tres años de formación - de la LPMT y en el ciclo de profundización - los últimos dos años. En estos ciclos se abordan diversos asuntos de la vida de los pueblos (ver figura 1 en las preguntas problematizadoras de los seminarios), a partir de preguntas como las siguientes: ¿cómo estamos en la actualidad, yo, en la familia, en la comunidad, en la región, en el país, en la Madre Tierra?; ¿cómo era la vida antes de la colonización?; ¿qué ha cambiado según la historia ancestral, según el territorio y la lengua?; ¿qué nuevos retos y posibilidades plantea a los pueblos la relación intercultural y con la madre tierra?

Estas preguntas se entretejen en encuentros educativos denominados: Seminario integrativo, Etnomatemáticas, Leguajes y Comunicación, Planes de Vida y Proyección Comunitaria. En el Cuadro 1 aparecen todas las especificaciones del núcleo básico del programa de formación de la versión en la que los dos profesores Gunadule se formaron que tiene como característica especial una orientación organizativa con base en problematizaciones de tipo indisciplinar:

Cuadro 1. Núcleo básico de la LPM

\begin{tabular}{|c|c|c|c|c|c|c|c|}
\hline & & & $\begin{array}{r}\text { Licenciatura er } \\
\text { - énfasis en s} \\
\text { Duración: } 10 \text { sem }\end{array}$ & $\begin{array}{l}\text { Pedag } \\
\text { alud con } \\
\text { estres (5 }\end{array}$ & $\begin{array}{l}\text { gía de la Ma } \\
\text { unitaria inte } \\
\text { años) - Tota }\end{array}$ & $\begin{array}{l}\text { dre Tierra } \\
\text { cultural } \\
180 \text { créditos }\end{array}$ & \\
\hline & & & & Núcleo $b$ & ásico (tres pr & imeros años) & \\
\hline & : & temáti- & Vida de los pueblos & y diálog & de saberes & $\begin{array}{l}\text { Formación peda } \\
\text { desde la intercultu }\end{array}$ & $\begin{array}{l}\text { gica } \\
\text { lidad }\end{array}$ \\
\hline & & & & Form & ación en inve & stigación & \\
\hline 1 & 18 & $\begin{array}{c}\text { Reen- } \\
\text { cuentro } \\
\text { con } \\
\text { nuestros } \\
\text { saberes y } \\
\text { nuestras }\end{array}$ & $\begin{array}{l}\text { Seminario Integral I } \\
\text { ¿Cómo estamos en } \\
\text { nuestra comunidad? } \\
\text { ¿Qué afecta nuestra vida } \\
\text { comunitaria, familiar y } \\
\text { personal? ¿Cómo la crisis } \\
\text { de nuestra vida personal, } \\
\text { familiar y comunitaria } \\
\text { afecta el equilibrio con la } \\
\text { Madre Tierra? } \\
\text { (6 CR) }\end{array}$ & $\begin{array}{l}\text { Plan de } \\
\text { Vida I } \\
(2 \text { CR })\end{array}$ & $\begin{array}{l}\text { Proyección } \\
\text { comunita- } \\
\text { ria I } \\
(3 \mathrm{CR})\end{array}$ & $\begin{array}{l}\text { Educación, lenguajes y } \\
\text { comunicación I } \\
(4 \mathrm{CR})\end{array}$ & $\begin{array}{l}\text { Etno- } \\
\text { Mate- } \\
\text { máticas } \\
\text { I } \\
(3 \mathrm{CR})\end{array}$ \\
\hline 2 & 18 & $\begin{array}{l}\text { realida- } \\
\text { des }\end{array}$ & $\begin{array}{l}\text { Seminario Integral II } \\
\text { ¿Cómo era la vida antes } \\
\text { en nuestra comunidad? } \\
\text { ¿Qué debemos rescatar, } \\
\text { qué debemos cambiar? } \\
\text { (6CR) }\end{array}$ & $\begin{array}{l}\text { Plan de } \\
\text { Vida II } \\
(2 \text { CR })\end{array}$ & $\begin{array}{l}\text { Proyección } \\
\text { comunitaria } \\
\text { II } \\
(3 \mathrm{CR})\end{array}$ & $\begin{array}{l}\text { Educación, lenguajes y } \\
\text { comunicación } \\
(4 \mathrm{CR})\end{array}$ & $\begin{array}{l}\text { Etno- } \\
\text { Mate- } \\
\text { máticas } \\
\text { II } \\
(3 \mathrm{CR})\end{array}$ \\
\hline
\end{tabular}

continua

\footnotetext{
${ }^{8}$ En el sentido presentado por Miguel, Vilela y Moura (2012) y problematizado desde la educación indígena por Tamayo-Osorio (2017a).
} 
Cuadro 1. continuación

\begin{tabular}{|c|c|c|c|c|c|c|c|c|c|}
\hline & & & $\begin{array}{r}\text { Licenci } \\
\text { - énfas } \\
\text { Duración: }\end{array}$ & $\begin{array}{l}\text { ura e } \\
\text { en s } \\
\text { sem }\end{array}$ & $\begin{array}{l}\text { Pedago } \\
\text { alud com } \\
\text { estres (5 }\end{array}$ & $\begin{array}{l}\text { gía de la Ma } \\
\text { unitaria inte } \\
\text { años) - Tota }\end{array}$ & $\begin{array}{l}\text { dre Tierra } \\
\text { cultural } \\
180 \text { créditos }\end{array}$ & & \\
\hline & & & & & Núcleo b & ásico (tres pr & imeros años) & & \\
\hline 吾 & : & $\begin{array}{l}\text { Ejes } \\
\text { temáti- }\end{array}$ & Vida de los $p$ & ieblos & y diálogo & de saberes & $\begin{array}{r}\text { Formaci } \\
\text { desde la ir }\end{array}$ & $\begin{array}{l}\text { pedag } \\
\text { ercultur }\end{array}$ & $\begin{array}{l}\text { gica } \\
\text { lidad }\end{array}$ \\
\hline & & & & & Form & ación en inve & stigación & & \\
\hline 3 & 18 & & $\begin{array}{l}\text { Seminario Inte } \\
\text { III ¿Cómo el pa. } \\
\text { presente colonial } \\
\text { nuestra vida e id } \\
\text { indigena? } \\
\text { (6 CR) }\end{array}$ & $\begin{array}{l}\text { ral } \\
\text { do y } \\
\text { ecta } \\
\text { tidad }\end{array}$ & \begin{tabular}{|l|} 
Plan de \\
Vida III \\
$(2 \mathrm{CR})$
\end{tabular} & $\begin{array}{l}\text { Proyección } \\
\text { comunitaria } \\
\text { III } \\
(3 \mathrm{CR})\end{array}$ & $\begin{array}{l}\text { Educación, len } \\
\text { comunicación } \\
\text { III } \\
(4 \mathrm{CR})\end{array}$ & rajes y & $\begin{array}{l}\text { Etno- } \\
\text { Mate- } \\
\text { máticas } \\
\text { III } \\
(3 \mathrm{CR})\end{array}$ \\
\hline 4 & 18 & $\begin{array}{l}\text { Diálogo } \\
\text { con los } \\
\text { otros }\end{array}$ & $\begin{array}{l}\text { Seminario Inte } \\
\text { IV ¿Cómo otros } \\
\text { indigenas y no in } \\
\text { del país, Améric } \\
\text { Latina y el mun } \\
\text { vienen enfrentano } \\
\text { efectos de la colon } \\
\text { y la globalización } \\
\text { (6 CR) }\end{array}$ & $\begin{array}{l}\text { ral } \\
\text { ueblos } \\
\text { genas } \\
\text { los } \\
\text { yación }\end{array}$ & $\begin{array}{l}\text { Plan de } \\
\text { Vida } \\
\text { IV } \\
(2 \mathrm{CR})\end{array}$ & $\begin{array}{l}\text { Proyección } \\
\text { comunitaria } \\
\text { IV } \\
(3 \mathrm{CR})\end{array}$ & $\begin{array}{l}\text { Educación, } \\
\text { lenguajes y } \\
\text { comunicación } \\
\text { IV } \\
\text { (4 CR) }\end{array}$ & $\begin{array}{l}\text { Com- } \\
\text { pe- } \\
\text { tencia } \\
\text { lectora } \\
\text { segun- } \\
\text { da len- } \\
\text { gua I } \\
(0 \text { CR })\end{array}$ & $\begin{array}{l}\text { Etno- } \\
\text { Mate- } \\
\text { máticas } \\
\text { IV } \\
(3 \mathrm{CR})\end{array}$ \\
\hline 5 & 18 & $\begin{array}{l}\text { El futuro } \\
\text { que } \\
\text { soñamos }\end{array}$ & $\begin{array}{l}\text { Seminario Inte } \\
\mathrm{V} \text { ¿Cómo queren } \\
\text { sea la vida en nu } \\
\text { comunidad? } \\
\text { ¿Qué proponemo } \\
\text { el cuidado de la I } \\
\text { Tierra? } \\
\text { (6 CR }\end{array}$ & $\begin{array}{l}\text { ral } \\
\text { s que } \\
\text { tra } \\
\text { para } \\
\text { adre }\end{array}$ & $\begin{array}{l}\text { Plan de } \\
\text { Vida V } \\
(2 \mathrm{CR})\end{array}$ & $\begin{array}{l}\text { Proyección } \\
\text { comunita- } \\
\text { ria } \mathrm{V} \\
(3 \mathrm{CR})\end{array}$ & $\begin{array}{l}\text { Educación, } \\
\text { lenguajes y } \\
\text { comunicación } \\
\text { V } \\
\text { (4 CR) }\end{array}$ & $\begin{array}{l}\text { Com- } \\
\text { pe- } \\
\text { tencia } \\
\text { lectora } \\
\text { se- } \\
\text { gunda } \\
\text { lengua } \\
\text { II } \\
(0 \text { CR })\end{array}$ & $\begin{array}{l}\text { Etno- } \\
\text { Mate- } \\
\text { máticas } \\
\text { V } \\
\text { (3 CR) }\end{array}$ \\
\hline 6 & 18 & & $\begin{array}{l}\text { Seminario } \\
\text { Integral VI. } \\
\text { Construcción } \\
\text { de propuestas } \\
\text { de aplicación } \\
\text { al Plan de } \\
\text { Vida desde una } \\
\text { perspectiva de } \\
\text { cuidado de la } \\
\text { Madre Tierra } \\
\text { (6CR) }\end{array}$ & $\begin{array}{l}\text { For- } \\
\text { ma- } \\
\text { ción } \\
\text { Ciu- } \\
\text { da- } \\
\text { dana } \\
(0 \\
\text { CR })\end{array}$ & $\begin{array}{l}\text { Plan de } \\
\text { Vida } \\
\text { VI } \\
(2 \mathrm{CR})\end{array}$ & $\begin{array}{l}\text { Proyección } \\
\text { comunitaria } \\
\text { VI } \\
(3 \mathrm{CR})\end{array}$ & $\begin{array}{l}\text { Educación, } \\
\text { lenguajes y } \\
\text { comunicación } \\
\text { VI } \\
(4 \mathrm{CR})\end{array}$ & $\begin{array}{l}\text { Com- } \\
\text { pe- } \\
\text { tencia } \\
\text { lectora } \\
\text { se- } \\
\text { gunda } \\
\text { lengua } \\
\text { III } \\
(0 \text { CR })\end{array}$ & $\begin{array}{l}\text { Etno- } \\
\text { Mate- } \\
\text { máticas } \\
\text { VI } \\
(3 \mathrm{CR})\end{array}$ \\
\hline
\end{tabular}

Fuente: Universidad de Antioquia (2006a, p. 43). 
En LPM hay un segundo ciclo de profundización (ver Cuadro 2) en el que cada estudiante indígena en formación, en acuerdo con su familia y comunidad, elige un campo de saber en el que conciba mayores posibilidades de contribuir con el mejoramiento de la vida escolar y comunitaria. Las líneas de profundización pueden ser: Lenguajes e Interculturalidad, Salud Comunitaria Intercultural, Ordenamiento y Autonomía Territorial (estos pueden variar por cohorte). Al comenzar este ciclo también se concreta la Práctica Pedagógica Profesional y el Trabajo de Investigación para Grado.

Cuadro 2. Núcleo de profundización LPM

\begin{tabular}{|c|c|c|c|c|c|c|c|}
\hline \multirow{3}{*}{ 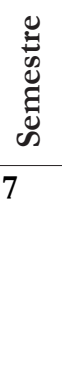 } & \multirow{3}{*}{ 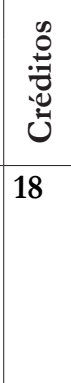 } & \multirow{3}{*}{$\begin{array}{l}\text { Ejes temáticos } \\
\text { Cuidado de la } \\
\text { salud en la } \\
\text { infancia }\end{array}$} & \multicolumn{5}{|c|}{ Núcleo de énfasis (dos últimos años) } \\
\hline & & & \multicolumn{3}{|c|}{$\begin{array}{l}\text { Vida de los pueblos y diálogo de saberes } \\
\text { (énfasis) }\end{array}$} & \multicolumn{2}{|c|}{$\begin{array}{l}\text { Formación pedagógica } \\
\text { desde la interculturali- } \\
\text { dad (énfasis) }\end{array}$} \\
\hline & & & $\begin{array}{l}\text { Saberes y } \\
\text { prácticas } \\
\text { ancestrales } \\
\text { I } \\
(4 \mathrm{CR})\end{array}$ & $\begin{array}{l}\text { Perspectivas } \\
\text { interculturales } \\
\text { I } \\
(4 \mathrm{CR})\end{array}$ & $\begin{array}{l}\text { Asuntos jurí- } \\
\text { dicos sobre la } \\
\text { salud } \\
\text { (3 CR) }\end{array}$ & $\begin{array}{l}\text { Etno- } \\
\text { Matemá- } \\
\text { ticas } \\
\text { VII } \\
(3 \mathrm{CR})\end{array}$ & $\begin{array}{l}\text { Pedagogías } \\
\text { desde la } \\
\text { diversidad } \\
\text { cultural I } \\
(4 \mathrm{CR})\end{array}$ \\
\hline 8 & 18 & $\begin{array}{l}\text { Cuidado de la } \\
\text { salud en la } \\
\text { adultez }\end{array}$ & $\begin{array}{l}\text { Saberes y } \\
\text { prácticas } \\
\text { ancestrales } \\
\text { II } \\
(4 \mathrm{CR})\end{array}$ & $\begin{array}{l}\text { Perspectivas } \\
\text { interculturales } \\
\text { II } \\
(4 \mathrm{CR})\end{array}$ & $\begin{array}{l}\text { Relaciones } \\
\text { intersectoriales } \\
\text { en el cuidado de } \\
\text { la salud } \\
\text { (3 CR) }\end{array}$ & $\begin{array}{l}\text { Etno- } \\
\text { Matemá- } \\
\text { ticas } \\
\text { VIII } \\
(3 \mathrm{CR})\end{array}$ & $\begin{array}{l}\text { Pedagogías } \\
\text { desde la diver- } \\
\text { sidad cultural } \\
\text { II } \\
\text { (4 CR) }\end{array}$ \\
\hline 9 & 18 & $\begin{array}{l}\text { Cuidado de la } \\
\text { salud en la } \\
\text { senectud }\end{array}$ & $\begin{array}{l}\text { Saberes y } \\
\text { prácticas } \\
\text { ancestrales } \\
\text { III } \\
\text { (4 CR)I }\end{array}$ & $\begin{array}{l}\text { Perspectivas } \\
\text { interculturales } \\
\text { III } \\
(4 \mathrm{CR})\end{array}$ & $\begin{array}{l}\text { Electiva I } \\
(3 \mathrm{CR})\end{array}$ & $\begin{array}{l}\text { Electiva } \\
\text { II } \\
(3 \mathrm{CR})\end{array}$ & $\begin{array}{l}\text { Pedagogías } \\
\text { desde la diver- } \\
\text { sidad cultural } \\
\text { III } \\
(4 \mathrm{CR})\end{array}$ \\
\hline 10 & 18 & $\begin{array}{l}\text { Cuidado de la } \\
\text { salud en la } \\
\text { familia, la co- } \\
\text { munidad y la } \\
\text { Madre Tierra }\end{array}$ & $\begin{array}{l}\text { Saberes y } \\
\text { prácticas } \\
\text { ancestrales } \\
\text { IV } \\
(4 \mathrm{CR})\end{array}$ & $\begin{array}{l}\text { Perspectivas } \\
\text { interculturales } \\
\text { IV } \\
(4 \mathrm{CR})\end{array}$ & $\begin{array}{l}\text { Electiva III } \\
(3 \mathrm{CR})\end{array}$ & $\begin{array}{l}\text { Electiva } \\
\text { IV } \\
(3 \mathrm{CR})\end{array}$ & $\begin{array}{l}\text { Pedagogías } \\
\text { desde la diver- } \\
\text { sidad cultural } \\
\text { IV } \\
\text { (4 CR) }\end{array}$ \\
\hline
\end{tabular}

Fuente: Universidad de Antioquia (2006a, p. 44).

De acuerdo con su énfasis, el licenciado estará en capacidad de desempeñarse en diversos campos de liderazgo en la propia comunidad que contribuyan para el plan de vida de su pueblo, en el caso de los profesores indígenas Gunadule uno de ellos opto por el eje Lenguajes e Interculturalidad y el otro por una formación en el eje de Salud Comunitaria Intercultural, posibilitando una formación pedagógica de la Madre Tierra con base en la Interculturalidad. 
En la LPMT los cursos de Etnomatemática se desarrollan en clases donde todos los estudiantes se reúnen, sin diferenciación por énfasis. Este curso se configura desde las siguientes dimensiones en el programa:

[...] conceptual, histórica, cognitiva, epistemológica, política y educativa, las cuales permiten considerar al conocimiento matemático como una producción cultural y social de los diferentes grupos y comunidades. A través de estas dimensiones se reconoce, por ejemplo, que la matemática bajo un perspectiva de la investigación, tiene un fin en sí misma, pero cuando está dirigida hacia la educación se deben establecer interacciones entre las diferentes prácticas y procedimientos que involucran conceptos matemáticos; en el aspecto político, el objetivo es el de denunciar y transformar las relaciones de poder que permean los procesos de validación y legitimación del saber; y en lo relacionado con el proceso pedagógico, el desafío está centrado en las posibilidades y estrategias de enseñanza y de aprendizaje que consideren el ambiente multicultural del aula de clase. (UNIVERSIDAD DE ANTIOQUIA, 2006a, p. 84).

La propuesta de incluir la Etnomatemática como parte de la formación pedagógica desde la interculturalidad puede ser entendida, desde nuestra perspectiva, como parte del proceso de deconstrucción del pensamiento occidental como un pensamiento abismal ${ }^{9}$ toda vez que en la LPMT, en estos cursos, se parte de la existencia de los dos lados - la Matemática y las matemáticaS - buscando posibilitar que ambos estén al mismo tiempo presentes para problematizar prácticas sociales, como centro de la formación de los futuros profesores indígenas, por ejemplo, problematización de prácticas de orientación espacial en los territorios.

Entendiendo que, hablar en matemáticaS como lo esclarece Tamayo-Osorio (2017b), es entender conjuntos de acciones normativamente orientadas con fines inequívocos, esto es, como juegos de lenguaje en una perspectiva wittgensteiniana.

\section{La Etnomatemática y sus efectos en la formación de dos profesores indígenas Gunadule}

Diversas Instituciones de Educación Superior de América Latina han incorporado dentro de sus programas curriculares la Etnomatemática como disciplina, con la finalidad de que dentro de las universidades los saberes y lenguas de los pueblos indígenas sean elementos fundamentales en el proceso de formación docente, y así, contribuir con la promoción de relaciones interculturales equitativas con la valoración de la diversidad cultural, puesto que

\footnotetext{
${ }^{9}$ Entendido como un "un sistema de distinciones visibles e invisibles, las invisibles constituyen el fundamento de las visibles. Las distinciones invisibles son establecidas a través de líneas radicales que dividen la realidad social en dos universos, el universo de "este lado de la línea" y el universo del “otro lado de la línea" (SANTOS, 2010, p. 11). La característica esencial del pensamiento abismal es la imposibilidad de la copresencia de los dos lados de la línea, desde nuestro punto de vista, en los programas de formación de profesores indígenas.
} 
la tarea de "interculturalizar toda la educación superior", de hacerla verdaderamente "universalista" y no monocultural subalternamente seguidora del legado europeo moderno e igualmente articulada al mercado mundial, sigue pendiente (Mato 2008b, 2008c). No obstante, producto de las luchas de los movimientos indígenas y afrodescendientes, así como de las de otros actores sociales latinoamericanos y extra-regionales con agendas transformadoras convergentes, en las últimas dos décadas se han establecido cerca de un centenar de IES y programas que de maneras muy diversas apuntan a resolver esta carencia. (MATO, 2011, p. 68).

Frente a estos retos de las universidades consideramos que la LPM surgió como un programa para reafirmar la interculturalidad como vía para la convivencia de epistemologías diferentes de forma horizontal. Para esto, la licenciatura parte de las cosmovisiones y cosmogonías de los pueblos como uno de los pilares fundamentales para entender la historia y ver hacia el futuro. De ese modo, la Etnomatemática como uno de sus componentes curriculares ha sido fundamental, ya que nos invita a ver la producción de conocimientos matemáticoS como culturales y sociales pues desde allí es donde se sustenta el pensamiento y conocimiento ancestral, en palabras del profesor Martínez Montoya ${ }^{10}$,

En los cursos de Etnomatemáticas aprendí más de la historia de origen, de nuestra manera de ver el mundo. Yo no sabia, por ejemplo, que en la lengua de nosotros no existe la palabra Matemática, esto lo aprendí con don Jaime en una investigación que bice con Richardy Carolina. Aprendí que hay semejanza entre la Matemática de la escuela con los conocimientos de nuestras prácticas que son denominados como Immal iddogedi igala gunadule ${ }^{11}$, pero que son diferentes.

El profesor nos cuenta cómo desde los estudios realizados en los cursos de Etnomatemática al investigar en, para, desde y con su comunidad, fue posible que él comenzara a identificar familiaridad entre formas de conocer y significar diferentes, la Matemática de carácter eurocéntrico que se trabaja en la escuela y Immal iddogedi igala Gunadule los conocimientos movilizados en sus prácticas sociales. Este tipo de interacción entre epistemologías de diferente naturaleza evidencia la posibilidad de diálogo intercultural que puede ser llevado a las escuelas desde la enseñanza de las matemáticaS en una perspectiva Etnomatemática, partiendo de las realidades que viven los niños y jóvenes indígenas. A respecto Olo Wintiyape ${ }^{12}$ nos dice lo siguiente ${ }^{13}$,

\footnotetext{
${ }^{10}$ Entrevista concedida a la investigadora por Martínez Montoya el 24 de enero de 2014.

${ }^{11}$ Immal significa cosa, objeto; iddogedi entender comprender; igala significa camino, método; gunadule persona, es decir, método de comprender qué es un objeto. Immal iddogedi igala gunadule es el camino que se sigue para comprender, identificar y organizar el conocimiento en una práctica social.

${ }^{12}$ En este texto usamos los nombres de los profesores según el Término de Libre Consentimiento para la investigación que fue concedido por ellos.

${ }^{13}$ Entrevista concedida a la investigadora por Olo Wintiyape el 7 de Julio de 2011.
} 
Para mi la experiencia en la LPMT fue excelente, porque teníamos la oportunidad de ir a la universidad y escuchar otros discursos, pero también haciamos trabajos comunitarios y visitar otras comunidades, y pasar por todos estos espacios, nos permitió darnos cuenta de que nuestra cultura posee otros conocimientos, que no tienen que ser vistos teniendo como parámetro los conocimientos de la Matemática occidental. Para mi fue un avance muy muy positivo, porque no solamente este trabajo sirve para mí, sino para todos... lo más importante para mi comunidad. Yo diría que si ustedes, como ya lo dije en otro momento, [refiriéndose a los investigadores de la universidad] no estwieran acompañándonos nosotros tampoco hiciéramos esas indagaciones a los sabios...

Así, los cursos de Etnomatemática aparecen como una respuesta a los diversos desafíos que desde las organizaciones indígenas y las comunidades se han colocado a nivel de educación universitaria, particularmente por su énfasis en retomar la sabiduría ancestral estableciendo relaciones con otros saberes matemáticoS, de tal forma que, la LPM ha posibilitado que los profesores, que enseñaran matemáticaS, en las escuelas indígenas, se formen en un marco claramente intercultural, pero también en procesos de orden pedagógico que inician con el fortalecimiento de su lengua materna, pues

La lengua es donde encontramos los secretos de nuestros pueblos indigenas, desde alli aprendí que somos poseedores de una sabiduría de muchos siglos atrás, que también está en las prácticas sociales que desarrollamos cada dia, y es alli donde están los conocimientos Immal iddogedi igala Gunadule no en la escuela, hay que escuchar a los sabios de alli parte todo. Hay que buscar formas para relacionarlas [la Matemática y los conocimientos Guna] y vimos que era posible hacerlo con el curso de Etnomatemáticas. ${ }^{14}$

Estas palabras de Olo Wintiyape nos remiten a los principios (UNIVERSIDAD DE ANTIOQUIA, 2006a) que orientan la formación del futuro profesor indígena que ensenará matemáticaS en la LPM, y que son los elementos fundamentales de la Pedagogía de la Madre Tierra desde donde es posible pensar una educación otra para los pueblos indígenas:

1 Silencio como posibilidad de escucharnos y de escuchar. El silencio como categoría pedagógica invita a mirar para dentro, invita a conocer de otro modo y reconocer que a través del silencio consciente han pervivido conocimientos y pueblos ancestrales; 2 La observación intencionada, la mirada que reconoce, crea y recrea la vida, las personas, la educación, la cultura. El observar desde el corazón, desde la historia, el observar para caminar. La observación con el corazón para aprender y acompañar;

$3 \mathrm{La}$ comunidad. Se promueven procesos formativos fundamentados en el diálogo estudiantes-comunidades a partir de sus mismas realidades y sus propias dinámicas. El principio comunitario implica asumir un papel como agente político de transformación, con valor por la historia y por la construcción intercultural equitativa;

\footnotetext{
${ }^{14}$ Entrevista concedida a la investigadora por Olo Wintiyape el 12 de septiembre de 2015.
} 
4 Intercultural. Desde la perspectiva de los pueblos subordinados (mestizos, indígenas, afros, campesinos, mujeres) por la dominación en el saber, en el ser, en el poder y en la naturaleza, partimos de la urgencia de construir relaciones de solidaridad, corresponsabilidad y mutuo respeto entre los pueblos sin desconocer las particularidades y valores propios;

5 Dialogo de saberes, de preguntas, de conocimientos y experiencias en un ejercicio de construcción colectiva donde la interacción se re-contextualiza y re-significa, posibilitando la reflexividad y la configuración de sentidos en los procesos, acciones, saberes, historias y territorialidades, ampliando, no solamente los niveles de comprensión de las realidades de las comunidades, sino también las posibilidades de las mismas;

6 Tejido con diferentes materiales y técnicas ha sido a través de la historia de la humanidad una de las expresiones culturales más permanentes. El tejido como principio pedagógico es comprendido como una práctica permanente material y del pensamiento de las culturas, el cual se construye y recrea, refleja la participación individual y colectiva. (UNIVERSIDAD DE ANTIOQUIA, 2006a).

Estos principios hacen posible incluir la Etnomatemática como provocadora de otras formas de conducirse en el mundo, que hacen visible y accesible la comprensión de otras historias no lineales, en espacios otros, donde la cultura y la naturaleza no pueden analizarse de forma dicotómica y donde las trayectorias de las comunidades posibilitan entender que una “[...] definición de cuáles contenidos son válidos y/o significativos en cada contexto, va a depender de la compresión de cuáles conocimientos deben ser apropiados como productos de la cultura y cómo estas experiencias seguirán construyendo nuevos conocimientos" (FERRI, 2002, p. 88).

En este sentido, y considerando la experiencia de los dos profesores Gunadule formados en la LPM, podríamos afirmar, que al problematizar prácticas socioculturales de forma indisciplinar (TAMAYO-OSORIO, 2017b) orientados desde, para y con las comunidades, amplia los horizontes de significación sobre las formas de conocer de culturas diferentes; además, se evidencia que los conocimientos matemáticoS movilizados en las prácticas, no pueden ser encajados en las áreas o disciplinas en las cuales tradicionalmente se ha distribuido el currículo, en este sentido el profesor Martínez Montoya ${ }^{15}$ manifiesta lo siguiente,

\begin{abstract}
Los contenidos de la Matemática de la escuela son otra cosa diferente de los conocimientos de las prácticas de mi pueblo, por eso, estos últimos, no están en el currículo. Pero bay que hacer esfuerzos para que desde la escuela defendamos la tradición milenaria que se viene trasmitiendo de generación a generación de los Guna desde la pedagogía de la Madre Tierra. Además, desde los cursos de Etnomatemática pude entender que el territorio y lenguaje son la base de todo, de alli viene el conocimiento propio de mi comunidad, y no se trata de compararlos con los contenidos de los currículos que llegan con la etnoeducación y sus libros guías, pues son producto de otra cultura, de otras tradiciones.
\end{abstract}

\footnotetext{
${ }^{15}$ Entrevista concedida a la investigadora por Martínez Montoya el 17 de enero de 2015.
} 
Por otro lado, pensamos que los cursos de Etnomatemática en el plan curricular de la LTPM dan cuenta de la importancia de incentivar a los futuros profesores indígenas, que enseñaran matemáticaS, para estudiar e investigar la propia cultura, y así comprender lo que se ha llamado de matemáticas ancestrales o matemáticas indígenas (HIGUITA RAMIREZ, 2014) como formas de trasgresión a la imposición colonial que la Matemática, concebida como disciplina, ha ejercido. Lo anterior puede identificarse en la tabla 1, en la cual en el curso de Etnomatemática se procura discutir desde las problemáticas de las comunidades diversos tópicos:

1 Reconocer e identificar el conocimiento matemático producido por las comunidades desde y para sus representaciones sociales;

2 Estudiar las diferentes formas sociales de ver las matemáticas en relación con las diversas formas de explicar el mundo;

3 Problematizar desde las propias comunidades los conocimientos matemáticos en dialéctica con las prácticas socioculturales como la pintura, las esculturas, tejidos en tela, cestería.

Estas problematizaciones, de tipo indisciplinar, que no están segmentadas por contenidos, aproximan a los futuros profesores indígenas para conocer sus tradiciones y conocer las de otros pueblos, al mismo tiempo que, en palabras del profesor Guna Martínez Montoya,

a cambiar la forma de pensar; a pensar cómo realizar y rescatar lo que tenemos dentro de la comunidad, porque en la comunidad hay muchas bibliotecas para investigar, que son los sabios nuestros, son como bibliotecas ambulantes para conocer lo que hacemos ante la vida y cómo lo hacemos. Yo como estudiante de Licenciatura en Pedagogía de la Madre Tierra me he transformado mucho. Ya no pienso como hace dos años atrás, porque he investigado, practicado y utilizado nuestra memoria ancestral, la de la comunidad, en la escuela; y cada día aprendo nuevas cosas de la vida. (TAMAYO-OSORIO, 2012, p. 111 , grifos del autor).

Los cursos de Etnomatemática han posibilitado abrir brechas descolonizadoras de pensamientos, lo que nos permite entender ese discurso como una contra conducta, como un discurso que emerge al cuestionar algunas de las principales estructuras del campo Matemático, en especial a la universidad y su poder de verdad única y absoluta, esto es, la Etnomatemática, como un discurso que se presenta como resistencia, que no niega a la Matemática, pero que busca nuevas formas de pensar ese saber (MONTEIRO; MENDES, 2015). Desde esta óptica, los seminarios de Etnomatemática ofrecidos en la LPMT aproximan a los futuros profesores indígenas de la comprensión de que las actividades matemáticaS no se realizan en única práctica social - la del matemático universitario -, que, como afirma D’Ambrosio (2008), con su carácter de infalibilidad, de rigor, de precisión, afirmó su presencia, excluyendo otras formas de pensamiento, principalmente por el hecho de ser un instrumento esencial y poderoso en el mundo moderno, ella, se presentó como un dios sabio, milagroso y más poderoso que las divinidades de otras culturas. 


\section{Reflexiones en abierto}

La formación de profesores en la LPMT considerando la Etnomatemática como un elemento central, amplia los horizontes para repensar Educación Matemática Indígena en las universidades con una perspectiva decolonial e intercultural, desde la cual se configuran currículos y pedagogías críticas para ver la enseñanza y el aprendizaje de las matemáticaS entrelazados a la pregunta ¿a quién enseñamos? Y de este modo centrar la mirada en contextos particulares movilizadores de conocimientos matemáticoS, esto es, en sujetos específicos, al mismo tiempo que nos aleja de la pregunta ¿qué enseñar?, centrada única y exclusivamente en el contenido, una visión disciplinar del conocimiento, así, como la escuela moderna se ha organizado.

La LPM es un ejemplo en la construcción de programas de formación indígena más apropiados para los pueblos indígenas, ya que al partir de sus necesidades se incorpora a las dinámicas de los pueblos en relación intercultural con la dinámica de otros pueblos, emerge un programa de formación docente donde la subjetividad es el plano de fondo y con ello el interés de reivindicar las luchas de los pueblos indígenas en Colombia, para que se valoren y legitimen otras formas de educación no disciplinar, que podríamos llamar de educación indisciplinar, esa que viene desde las propias prácticas educativas de los pueblos, que considera las particularidades e intereses de cada una de las culturas.

Como efecto se transgreden líneas de formación de profesores indígenas, que enseñan matemáticas desterritorializando a la Matemática eurocéntrica en la escuela y territorializandola en otros términos como parte de la decolonialidad del saber y de las apuestas por otras educaciones. Lo anterior como parte del conjunto de demandas políticas y reclamaciones de autonomía que, desde el inicio del siglo XX hasta hoy, vienen planteando al Estado colombiano los grupos étnicos y sus organizaciones.

Es importante resaltar que este tipo de propuestas si bien surgen como innovadoras en un contexto universitario donde todo se presenta de forma disciplinar, es necesario que se hagan más comunes, propuestas estas, que partan del diálogo con las comunidades y que se acoplen a los tiempos y rituales de las comunidades, como Higuita Ramirez (2014) ya señaló.

La Licenciatura en Pedagogía de la Madre Tierra y sus principios pensados en los cursos de Etnomatemáticas, nos han llevado a conocer otras formas de ser y pensar que pueden dialogar de forma unilateral con los conocimientos Matemáticos occidentales, donde la interculturalidad no es un problema, al contrario, es uno de los caminos más eficaces para que nuestras sociedades logren sanar las brechas que nos han separado, unos de otros, por la imposición de un modelo único - de cultura, de Universidad, de escuela, de Matemática... - basado en la diferencia cultural, de creencias, de prácticas, de raza, de género... - y no en la diversidad.

La experiencia de los dos profesores Gunadule nos permitió ver algunos efectos de incluir la Etnomatemática en la LPM, en la medida en que se reconocen las raíces culturales e históricas de los conocimientos de sus pueblos como centro de todo el proceso formativo, además, porque los conocimientos que emergen en las problematizaciones indisciplinares están relacionados con la búsqueda de respuestas a problemas que se presentan en las necesidades de las prácticas sociales.

Así, la LPMT da cuenta de la necesidad de los pueblos indígenas al formar profesores que cuestionen y problematicen desde sus propias realidades la escuela como una institución que ha postergado la dominación de culturas que buscan la hegemonía mediante el conocimiento. 
En este sentido la Etnomatemática en la LPM permite "[...] entender el saber/hacer matemático a lo largo de la historia de la humanidad, contextualizado en diferentes grupos de interés, comunidades, pueblos y naciones" (D’AMBROSIO 2008, p. 17), lo que significa algo más que hacer un reconocimiento del saber/hacer matemático en los pueblos, se trata de entender lo que en estos pueblos se produce como conocimientos matemáticoS en relación con los desarrollos históricos, sociales, políticos, económicos y culturales propios de un grupo humano.

Por otro lado, es necesario apuntar que no debemos establecer patrones de comparación o traducción entre los conocimientos que llegan por medio de la escuela - el de la cultura occidental - a las comunidades indígenas, con los que son producidos, validados y legitimados en sus prácticas sociales. De lo que se trata es de entender el saber/hacer producido allí, en cada pueblo, desde sus dinámicas, desde sus lógicas. La validación de este conocimiento solo tiene sentido en la cultura en la que se desarrolla. En este sentido, oímos de la voz de Olo Wintiyape: "[...] todas las prácticas sociales tienen significados para nuestra comunidad". ${ }^{16}$

Finalmente creemos que pensar la formación de profesores indígenas, que enseñan matemáticaS, como una propuesta para la reivindicación política, cultural y académica, implica no solo descolonizar la mirada de la universidad sobre las prácticas sociales de estos pueblos, sino también aproximarnos a otras epistemologías y formas de organizar y pensar ser/hacer en el mundo, lo cual ha sido posible en la LPM al incluir la Etnomatemática en su programa, pues se han abierto espacios dialógicos en los que la Universidad y las comunidades ancestrales, juntas, cuestionen los paradigmas de formación de profeses indígenas, y no indígenas, que enseñaran matemáticaS, cargados de un carácter colonial, en cuyo marco se impusó como único y válido el conocimiento Matemático académico.

\section{Agradecimientos}

A la comunidad Guna de Alto Caimán por haberme acogido y a los maestros por permitirme haber sido partícipe de sus procesos de formación en la LTP. Igualmente a la Licenciatura en Pedagogía de la Madre Tierra por la acogida y las puertas que me han abierto durante estos años de trabajo colaborativo. Además, a la Coordenação de Aperfeiçoamento de Pessoal de Nível Superior (Capes) por la financiación de mis estudios doctorales de los cuales emerge este artículo.

\section{Referencias}

BLANCO ÁLVAREZ, H. La integración de la etnomatemática en la etnoeducación. In: ENCUENTRO COLOMBIANO DE MATEMÁTICA EDUCATIVA, 9., 2008, Valledupar. Memorias... Disponible en: < http:/ / funes.uniandes.edu.co/874/1/11Conferencias.pdf>. Accedido el: 21 ago. 2018.

\footnotetext{
${ }^{16}$ Entrevista concedida a la investigadora el 29 de julio de 2011.
} 
Licenciatura en Pedagogía de la Madre Tierra, etnomatemática y formación ...

CAMACHO, J. Entre lo local y lo global: perspectivas antropológicas en educación.

Repertorio Americano, San Jose, n. 7, p. 12-21, 1999.

CASTILLO, E.; ROJAS, A. Educar a los otros: estado, políticas educativas y diferencia cultural en Colombia. Popayán: Universidad del Cauca, 2005.

COLOMBIA. Constitución política de Colombia 1991. Disponible en: <http:// es.presidencia.gov.co/normativa/constitucion-politica>. Accedido el: 21 ago. 2018.

COLOMBIA. Decreto 1142 de junio 19 de 1978. Por el cual se reglamento el artículo 11 del decreto ley 088 de 1978 sobre educación de las comunidades indígenas. Disponible en: $<$ http://observatorioetnicocecoin.org.co/cecoin/files/Decreto_1142_1978.pdf>. Accedido el: 21 ago. 2018.

COLOMBIA. Decreto número 804 de 1995 (mayo 18): por medio del cual se reglamenta la atención educativa para grupos étnicos. Diario Oficial, Bogotá, n. 41853, 18 mayo 1995. Disponible en: <https://www.mineducacion.gov.co/1759/articles-103494_archivo_pdf. pdf>. Accedido el: 21 ago. 2018.

COLOMBIA. Ley 115 de febrero 8 de 1994. Por la cual se expide la ley general de educación. Bogotá, 1994. Disponible en: <https://www.mineducacion.gov.co/1621/ articles-85906_archivo_pdf.pdf $>$. Accedido el: 21 ago. 2018.

COLOMBIA. Departamento Administrativo Nacional de Estadística. La visibilización estadística de los grupos étnicos colombianos. Bogotá, [2005]. Disponible en: <https:// www.dane.gov.co/files/censo2005/etnia/sys/visibilidad_estadistica_etnicos.pdf>._Accedido el: 21 ago. 2018.

CUÉLLAR LEMOS, R. N. Nabba nana gala burbaba nanaedi igala odurdagge gunadule durdagedi nega gine: igal dummadi maidi sabbimala soganergwa naggulemaladi [La pedagogía de la Madre Tierra en una escuela indígena Gunadule: un estudio sobre la sabiduría de seis plantas de protección]. 2017. Tesis (Maestría en Educación) - Facultad de Educación, Universidad de Antioquia, Medellín, 2017.

CUÉLLAR LEMOS, R. N.; MARTÍNEZ MONTOYA, F. La revitalización del lenguaje de la medicina ancestral a partir de las veinticuatro variedades de plátano: una posibilidad para pensar una otra educación Gunadule desde la pedagogía de la Madre Tierra. 2013. Trabajo de pregrado (Licenciatura em Pedagogía) - Universidad de Antioquia, Medellín, 2013.

D'AMBROSIO, U. Ethnomathematics and its place in the history and pedagogy of mathematics. In: POWELL, A.; FRANKENSTEIN, M. (Ed.). Ethnomathematics: challenging eurocentrism in mathematics education. Albany: State University of New York, 1997. p. 13-24.

D’AMBROSIO, U. Etnomatemática: entre las tradiciones y la modernidad. México: Limusa. 2008.

D'AMBROSIO, U. Etnomatemática: um programa. Educação Matemática em Revista, Blumenau, v. 1, n. 1, p. 5-11, 1993. 
FERRI, C. Currículo multicultural: refletindo a educação escolar indígena. Contrapontos, Itajaí, v. 2, n. 1, p. 82-99, 2002.

GREEN, A.; SINIGUI, S.; ROJAS, A. Licenciatura en pedagogía de la Madre Tierra: una apuesta política, cultural y académica desde la educación superior y las comunidades ancestrales. In: RELACIONES interculturales en la diversidad. Córdoba: Universidad de Córdoba, 2013. p. 85-94.

HIGUITA RAMIREZ, C. La movilización de objetos culturales desde las memorias de la práctica de construcción de la vivienda tradicional Embera Chamí: posibilidades para pensar el (por)venir de la educación (matemática) indígena. 2014. Disertación (Maestría en Educación) - Universidad de Antioquia, Medellín, 2014.

JARAMILLO, D. La educación matemática en una perspectiva sociocultural: tensiones, utopías, futuros posibles. Revista Educación y Pedagogía, Medellín, v. 23, n. 59, p. 13-36, 2011.

MATO, D. Actualizar las propuestas de la reforma universitaria de 1918: interculturizar la educación superior: experiencias, avances y desafíos. Integración y Conocimiento, Córdoba, v. 2, n. 5, p. 35-52, 2016.

MATO, D. Universidades indígenas de América Latina: logros, problemas y desafíos. Revista Andaluza de Antropología, Sevilla, n. 1, p. 63-85, 2011.

MIGUEL, A.; VILELA, D. S.; MOURA, A. R. L. Problematização indisciplinar de uma prática cultural numa perspectiva wittgensteiniana. Reflexão e Ação, Santa Cruz do Sul, v. 20, n. 2, p. 6-31, 2012.

MONTEIRO, A.; MENDES, J. R. Etnomatemática como movimento de contraconduta na mobilização de saberes em práticas culturais. In: SIMPÓSIO INTERNACIONAL DE PESQUISA EM EDUCAÇÃO MATEMÁTICA, 6., 2015, Recife. Anais... Pirenópolis, 2015. p.1-11.

ORGANIZACIÓN NACIONAL DE LOS PUEBLOS INDÍGENAS DE LA

AMAZONIA COLOMBIANA. Denuncia pública por el derecho a la educacion propia de los pueblos indigenas de Colombia. [2017]. Disponible en: <https://opiac.org.co/denunciapublica-por-el-derecho-a-la-educacion-propia-de-los-pueblos-indigenas-de-colombia/> . Accedido el: 21 ago. 2018.

PANCHO, A. Educación superior indígena en Colombia: una apuesta de futuro y esperanza. Cali: Universidad de San Buenaventura, 2004.

ROJAS, A. ¿Etnoeducación o educación intercultural?: estudio de caso sobre la licenciatura en etnoeducación de la Universidad del Cauca. In: MATO, D. (Coord.). Diversidad cultural e interculturalidad en educación superior: experiencias en América Latina. Caracas: IESALC-UNESCO, 2008. p. 233-242.

ROJAS, A.; CASTILLO, E. Educar a los otros: estado, políticas educativas y diferencia cultural en Colombia. Popayán: Editorial Universidad del Cauca, 2005. 
SANTOS, B. S. Para descolonizar occidente: más allá del pensamiento abismal. Buenos Aires: Prometeo, 2010.

SIERRA, Z. (Ed.). Voces indígenas universitarias: expectativas, vivencias y sueño. Medellín: Universidad de Antioquia, 2004.

TAMAYO-OSORIO, C. (Re)significación del currículo escolar indígena, relativo al conocimiento [matemático], desde y para las prácticas sociales: el caso de los maestros indígenas Dule de la comunidad de Alto Caimán. 2012. (Maestría en Educación) Universdidad de Antioquia, Medellín, 2012.

TAMAYO-OSORIO, C. A colonialidade do saber: um olhar desde a educação matemática. Revista Latinoamericana de Etnomatemática, San Juan de Pasto, v. 10, n. 3, p. 39-58, 2017b.

TAMAYO-OSORIO, C. Vení, vamos hamacar el mundo, hasta que te asustes: uma terapia do desejo de escolarização moderna. 2017. Tese (Doctorado en Educación) Universidade Estadual de Campinas, Campinas, 2017a.

UNIVERSIDAD DE ANTIOQUIA. Información institucional sobre la licenciatura en etnoeducación. Disponible en: <http://www.udea.edu.co/portal/page/portal/portal/b. EstudiarUdeA/a.Pregrado/a.ProgramasPregrado>. Accedido el: 3 ago. 2014.

UNIVERSIDAD DE ANTIOQUIA. Propuesta de creación de programa académico: licenciatura en pedagogía de la madre tierra. [2006a]. Disponible en: < https:// zaydasierraudea.files.wordpress.com/2013/08/licenciatura-pedagogc3ada-madre-tierraintegrado-7jul2010f.pdf>. Accedido el: 20 jul. 2017.

UNIVERSIDAD DE ANTIOQUIA. Plan de desarrollo Universidad de Antioquia 2006-2016. Medellín: Editorial Universidad de Antioquia, 2006b.

UNIVERSIDAD DE ANTIOQUIA. Propuesta para la creación del programa de educación indígena de la U. de Antioquia. Resolución Académica 1752 del 18 de agosto de 2005. Disponible en: <https://zaydasierraudea.files.wordpress.com/2013/08/propuestacreacic3b3n-programa-educacic3b3n-indc3adgena-25may2005.pdf $>$. Accedido el: 3 ago. 2014.

Artigo recebido em 24/01/2018. Aceito em 11/03/2018.

Contato: Universidade Federal de São Carlos, Departamento de Metodologia de Ensino, Rod. Washington Luis, km 235, São Carlos 13565-905, Brasil. 